we should say that probably many of these would still have recovered if they had not been trephined. However this may be, such experience as theirs affords important evidence on the risks of the operation itself apart from any other injury.

\section{Clinical a dectures:}

\section{DISEASES OF THE SPINAL CORD.}

\author{
BX PROFESSOR CHARCOT, \\ PHYSTCIAN TO LA SALPÉTRIÈRE HOSPITAL.
}

\section{LECTURE II.}

\section{SYMPTOMATOLOGY OF LATERAL AMYOTROPHIC SCLEROSIS.}

IV. THE form of amyotrophic paralysis with which we are occupied most commonly manifests itself first in one of the upper limbs, so as to present a likeness to what is called "cervical paraplegia." Though the disease may date from only four, five, or six months, or a year at the utmost, wasting will have already attained a degree which, in protopathic muscular atrophy, is only observed at an advanced stage-perhaps five or six years from the beginning of the disease.

Things may remain in this state during two, six, or nine months, seldom more; after which the lower extremities are affected in turn, and generally in a manner quite different from the upper extremities. In the origin it is still paresia which makes its appearance, preceded, and accompanied for some time, by formication and numbness in the limb. But here paresia presents this notable feature, that it does not necessarily bring on muscular atrophy, as it does in the first case. On the contrary, the muscles may preserve, until the last stages of the disease, a fleshy appearance and a consistence which present a marked contrast to the condition of the upper limbs. This paraplegia offers this first particular feature, that it is not complicated with paralysis of the bladder or rectum, and that there exists no tendency to the formation of eschars. It is furthermore distinguished, as you will see, by other important charasters. Difficulty in the movements of the lower limbs makes rapid progress. The patient feels his legs heavy, and with difficulty moves them from the floor. In a short time he can walk only by the help of two supports. At last, it is impossible for him to stand on his legs, and then he is confined to bed, or obliged to sit the whole day in an arm-chair. When things have come to this point, a new and interesting phenomenon has already more or less shown itself : I mean permanent stiffness, or, in other words, spasmodic contraction of the muscles deprived of voluntary movement. For some time previously the patient will have noticed that, whether in bed or sitting, his lower limbs would occasionally extend or bend independently of his will, and for a few moments preserve the attitude thus involuntarily taken.

Extension is the most common fact in these cases, and it may be marked to the degree of determining almost tetanic stiffness, causing the lower limbs to be like rigid bars, susceptible only of being moved about en bloc. In addition to this, the limbs are sometimes agitated by convulsive tremulonsness. The stiffness becomes still more marked when the patient, supported by two assistants, attempts to get up and walk. Then the lower extremities stiffen to an excess during extension and adduction, at the same time that the feet assume the attitude of talipes varus equinus. This stiffness, which is of ten most marked, and occasionally less so, and which affects all the joints of the limbs through the spasmodic action of the muscles, together with the tremulousness which mostly accompanies it, renders walking and standing impossible. That which at first was only a transitory phenomenon is converted in a short time into a permanent symptom. Muscular rigidity persists without intermission in the flexor as well as the extensor muscles, although it predominates in the latter. It becomes equally difficult to excite forcible flexion of the extended limbs and forcible extension of the flexed limbs. Generally, at this time, if you lift up the end of the extended foot, you produce more or less durable tremulousness in the whole of the limb.

Thus, gentlemen, motor impotence is less linked with weakening of innervation than with the spasmodic state of the muscles. In these latter, moreover, nutrition, fur a long time, is accomplished in a normal manner. It is only after a long lapse of time that you see the muscles affected with fibrillar movements, and become atrophied throughout their extent, as in the upper extremities. Usually, when this atrophy reaches a certain degree the stiffness diminishes, though it never disappears entirely.

The early invasion of the lower limbs, and the nature of the phenomena of which they are the seat, is a distinctive feature, standing out in contrast with what we know of primary spinal amyotrophy, in which, as you remember, the lower limbs are affected only during the last stages of the disease. They constitute, so to say, the characters of a second stage; the third one being marked, as we are going to see, by the appearance of bulbar phenomena.

$\nabla$. The appearance of these last symptoms is in some way obligatory. Until now they have never been absent. They take in the phenomena which, by their combination, make up the syndroma designated by the name of labio-glossolaryngeal paralysis. We will only allude, in passing, to this phase of the disease, as it is a subject to which we must return when we treat in particular of paralysis depending on a bulbar cause.

I will only mention, so as not to omit altogether some of the most interesting features of the picture, the following symptoms:-

1. Paralysis of the tongue, inducing difficulty of swallowing, and difficulty of articulating words, amounting sometimes to total loss of speech. The paralysed tongue soon presents, in the greater number of cases, a certain degree of atrophy; it looks shrunk, as if wrinkled, and is agitated by vermicular movements.

2. Paralysis of the soft palate, which gives a nasal sound to speech, and combines with the laryngeal paralysis in rendering deglutition difficult.

3. Paralysis of the orbicularis oris, the chief consequence of which is modification of the features of the face. The mouth is considerably enlarged through the predominating action of the unaffected muscles of the face. The naso-labial grooves are very much marked. These various symptoms give to the physiognomy a whining expression. Occasionally, the mouth remains permanently half open, and permits of an incessant dribbling of viscous saliva.

4. Lastly, on account of the involvement of the first nuclei of the pneumogastric, grave disturbance of respiration and circulation supervenes and brings on the death of the patient, already weakened long before by insufficient nourishment.

I am going to attempt, gentlemen, to sum up in a few features the symptomatologic characters of lateral amyotrophic sclerosis, considered in what we may call its normal conditions.

1. Paresia without anæsthesia of the upper limbs, accompanied by rapid wasting of the ensemble of the muscular groups, and preceded occasionally by numbness and formication. Spasmodic rigidity invades at a given moment the paralysed and atrophied muscles, and determines permanent deformity through contraction.

2. The lower limbs are involved in turn. Paresia, at first unattended by anæsthesia, affects them, and progresses rapidly, so as soon to render standing and walking impossible. To these symptoms is added spasmodic stiffness, first intermittent, then permanent, and complicated sometimes by spinal tonic epilepsy. The muscles of the paralysed limbs only become atrophied after a lapse of time, and never to the same degree as those of the upper limbs. The bladder and rectum are never affected. There does not exist any tendency to the formation of eschars.

3. The third stage is constituted by the aggravation of the preceding symptoms and by the appearance of bulbar symptoms. 
The three stages succeed each other in a short space of time. Six months to a year after the onset all the symptoms have accumulated, and are more or less strongly marked. Death arrives after two or three years, on an average, through the agency of the bulbar symptoms.

Such is the rule. Of course there exists the chapter of anomalies. These are, however, exceedingly few, and do not alter any of the essential outlines of the picture $I$ have just drawn. Thus the disease in certain cases begins in the lower limbs; in other cases it confines itself in the beginning either to one upper limb or one lower limb. Occasionally it remains limited for some time to one side of the body, in the form of hemiplegia. Lastly, in two cases the disease broke out with bulbar symptoms. But these, I repeat, are only accessory modifications; the ensemble of characteristic symptoms does not fail to be soon constituted.

The prognosis so far is exceedingly gloomy. There does not exist, I believe, a single case in which, the existence of all the above symptoms being combined, recovery ever took place. Is this verdict a definitive one? Only time can show.

VI. It remains, gentlemen, for me to bring together the lesions and the symptoms, and, in an investigation of pathological physiology, to seek the link which connects them all.

1. The paresia which comes on from the onset and the permanent contractiong which soon follow are incontrovertibly dependent upon lateral and symmetrical sclerosis.

I will remind you that wherever you meet with lateral sclerosis, contraction is sure, sooner or later, to show itself in a more or less marked degree. Thus: $(a)$ in sclerosis in disseminated patches; $(b)$ in cerebral hemiplegia with consecutive descending sclerosis; $(c)$ in transverse myelitis through compression, or spontaneous, when lateral descending degeneration is the result; $(d)$ lastly, in primary sclerosis of the lateral fasciculi.

2. Paresia and contraction precede atrophy. This fact is clinically establisher. We must needs, therefore, admit that lateral sclerosis, upon which they depend, takes place before the lesions of the anterior grey substance to which amyotrophy incontrovertibly attaches. Through what mechanism does the lesion of the grey substance join that of the white fascicles? Is it simply a case of propagation by extension, going on gradually? It is much more likely that the propagation is effected through the agency of the nervous filaments, which normally establish, as you know, a communication between the lateral fascicles and the anterior horns.

3. The system of the lateral fascicles has a tendency to be affected in its whole extent, and that very rapidly. But the lesion does not invade its totality all at once. Thus, as far as we can judge from clinical observations, it first involves the department which is in physiological connexion with the movements of the upper limbs. At a later period it reaches the department connected with the lower ones. Lastly, the group of cerebro-bulbar fascicles is invaded in turn.

It is remarkable that the alterations enumerated in the first and third sections very quickly reach the corresponding portions of the grey substance. Thus, the muscles of the tongue, and those of the upper limbs especially, begin to waste very shortly after the appearance of the paresic symptoms. It is not so in respect to the system connected with the lower limbs; as in these last, the paresia and contraction persist for a long time before amyotrophy accompanies them. These are particularities which we can only point out, without endeavouring for the present to venture upon any plausible explanation.

[Note-The patient referred to in Lecture 1 (see THE EANCET, p. 73) as being then under observation at La Charité has since died. Bulbar symptoms carried him off. The post-mortem was performed by M. Voisin, housesurgeon in the wards of M. Woillez. Examination of the spinal cord, made by M. Gombault, curator of the Faculty, lecturer on morbid anatomy, showed the existence of lateral symmetrical sclerosis, with atrophy of the motor nervous cells in the anterior horns of the cervical region of the cord and in the first nuclei of the bulbar nerves. The anatomical preparations relating to this case were shown to the pupils during a lecture on morbid anatomy at the Faculty of Medicine.]

\section{CALCULUS IMPACTED IN A VESICAL SAC; OPERATION ; RECOVERY.}

By SIR HENRY THOMPSON, F.R.C.S.,

SURGEON EXTRAORDINARY TO H.M. THE KING OF THY BRLGIANS, NURGEON AND PROEESSOR OF CLXNICAL SURGBRY TO UNIVERSITY COLLEGE HOSPITAL, RTC.

Cases in which a vesical calculus is impacted in a sac situated in or extending through the walls of the bladder are so rare that $I$ desire to place on record the following example which has recently occurred in my practice.

I saw Mrs. - , a lady aged fifty-two years, for the first time in June last, with symptoms which made it necessary to sound her. I did so, and found easily and distinctly enough a vesical calculus. I proposed therefore to remove it by any method which should appear to me to be the most appropriate, when, subsequently, under the influence of chloroform, I should ascertain more precisely its size and nature.

Accordingly, on July 6th, the patient having been rendered insensible by Mr. Clover, 1 introduced a lithotrite, and at once felt the stone on the left side of the bladder (patient's left), but, on attempting to seize it, failed readily to do so. Examining it more deliberately with the litho. trite, I soon learned that it was fixed in some unusual manner. I then withdrew the instrument and slowly carried my index finger through the urethra-often a better and safer mode of dilating the female urethra than any metallic dilator offers, - and found the calculus about one-third exposed in the bladder, and two-thirds contained in a sac and lying outside the cavity of the bladder. A finger in the vagina traced the outline and indicated its size. These con. ditions were verified in the same way by Mr. Foster and Mr. Clover, the only persons present. Reintroducing my finger, I insinuated my nail within the margin of the sac, and in about two minutes, by carefully working the finger round the stone, succeeded in turning it out of its bed entire into the cavity of the bladder, whence it was readily removed by the forceps after a slight incision in the left side of the urethra.

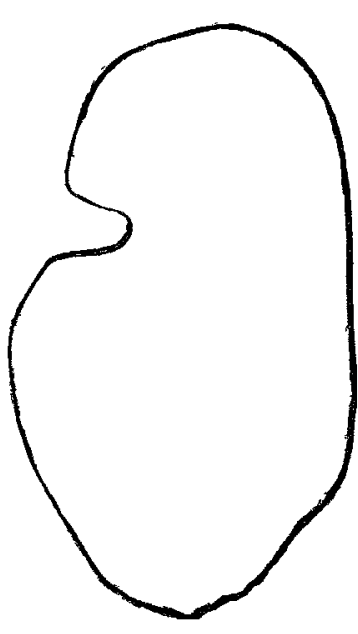

The annexed outline represents the stone of its exact size and form, the deep indentation corresponding with the neck of the sac, the larger portion of the stone being that which was contained within it, while the smaller was felt bare but immovable in the cavity of the bladder.

I have before operated on two cases of impacted vesical calculus in the male, and have seen two or three cases with others; but this is the first I have met with in the female, in whom they must be extremely rare, since the condition of the bladder which leads to the formation of vesical sacs-namely, obstruction at the neck, leading to protrusion and dilatation of the mucous membrane between the interlacing bands of muscular fibres-can very rarely exist; while this condition is, on the contrary, common in the male.

A rather free oozing of blood persisted during nearly a week, and more pain than usual in the act of micturition. The former has disappeared some days (July 25th); the latter diminishes gradually. The patient retains urine three or four hours, and has had no incontinence whatever.

Wimpole-street.

THE mortality in London last week was at the rate of 25 per 1000 annually. The deaths, 1640 in number, included 52 from scarlet fever, 31 from measles, 2 from small-pox, 4. from diphtheria, 20 from whooping-cough, 26 from different forms of fever, and 389 from diarrbœa. The heavy fatality from the latter disease occurred mainly among the infantile population, no less than 334 of the deaths being of infants under one year of age. 